\title{
Swelling behavior of poly (2-hydroxyethyl methacrylate) copolymer gels
}

\author{
S.M. Chabane Sari ${ }^{1, a}$, M. Benmouna ${ }^{1}$, M. Mahlous ${ }^{2}$ and M. Kaci ${ }^{3}$ \\ ${ }^{1}$ Macromolecular Research Laboratory Faculty of Sciences, University of Tlemcen, BP. 119, 13000, Algeria \\ ${ }^{2}$ Division of Nuclear Applications, Nuclear Research Center of Algiers, BP. 399 Alger-RP 16000, Algiers, Algeria \\ ${ }^{3}$ Organic Materials Laboratory, University A. Mira, 06000 Bejaïa, Algeria
}

\begin{abstract}
Hydrogels based on 2-hydroxyethyl methacrylate/dimethyl-aminoethyl methacrylate copolymers were prepared by gamma radiation-inducedco-polymerization at low temperature $\left(-78^{\circ} \mathrm{C}\right)$. The swelling behavior of hydrogels was studied by immersion of the polymer discs in buffered solutions at $\mathrm{pH}$ from 2 to 10 . The hydration process was followed gravimetrically by measuring the water uptake of the discs as a function of time. The results obtained have shown that the swelling behavior is reversible and depends on the polymer nature. Moreover, polymeric discs exhibited a good stability after repeating cycles of hydration in different buffer solutions. Scanning electron microscopy analysis reveals that hydrogel porosity can be controlled.
\end{abstract}

\section{INTRODUCTION}

During the past decades, most of the pharmaceutical research activities have focused on the synthesis of novel drugs and drug delivery systems. In this context, controlled release drug delivery systems (DDS) have a majorposition [1,2]. Among polymersystems that have been used as drug containers or release rate controlling barriers, hydrogels gained considerable interest and reviewed from different points of view [3,4]. Hydrogels consist ofa unique class of macromolecular networks that may absorb a large fraction of aqueous solvent. They are particularly suitable for biomedical and tissue engineering applications [5,6] because of their ability to simulate biological tissues [7]. They are used in a variety of applications in bioseparations, agriculture and enhanced oil recovery [8]. The hydrophilicity of the network is due to the presence of chemical residues suchas hydroxylic $(-\mathrm{OH})$, carboxylic $(-\mathrm{COOH})$, amidic $(-\mathrm{CONH}-)$, primary amidic $\left(-\mathrm{CONH}_{2}\right)$, sulphonic $\left(-\mathrm{SO}_{3} \mathrm{H}\right)$, and other groups that can be inserted in the polymer backbone or as lateral chains.

Hydrogels are polymer composites exhibiting a threedimensional network structure with the ability to swell and retain a considerable amount of water until $90 \%$ in its structure without dissolving [9].For this reason, a wide variety of hydrogels, either synthetic or natural, show an excellent behavior of biocompatibility [10], thus become very attractive for biomedical and pharmaceutical applications and for producing biosensors. They are used as supports for immobilization of bioreceptors aiming at determining the presence of moleculesand biological agents in a complex medium [11-13] as well as their concentrations.

Hydrogels of poly (2-hydroxyethyl methacrylate), henceforth designed as PHEMA, represent a system of low toxicity well accepted by body tissues and used as biomedical materials in various devices $[14,15]$. They are also known as implantable devices since fulfilling all the requirements of biocompatibility [16]. Several polymer

a e-mail: sm_chabanesari@yahoo.fr hydrogels exhibit a marked swelling-deswelling behavior in response to environmental changes including solvent composition, ionic strength, $\mathrm{pH}$, temperature [17], electric field and light [18]. For controlled delivery applications, hydrogels are particularly suitable to deliver entrapped either high or low molecular weight molecules such as drugs, pesticides and other bioactive agents in aqueous media simply by controlling the degree of cross-linking and extend of swelling [19].

In the present paper a number of polymer matrices were prepared from 2-hydroxyethyl methacrylate (HEMA), 2-hydroxyethyl acrylate (HEA) to which N,Ndimethylaminoethyl methacrylate (DMAEMA), Glycydylmethacrylate (GMA) were added as copolymers and trimethylolpropanetrimethacrylate (TMPTMA) as a crosslinker agent. We have studied their dynamic swelling behavior and degree of swelling at different $\mathrm{pHs}$.

\section{MATERIALS AND METHODS}

\subsection{Materials}

The monomers: 2-hydroxyethyl methacrylate (HEMA), 2-hydroxyethyl acrylate (HEA), N, N-dimethylaminoethyl methacrylate (DMAEMA), trimethylolpropanetrimethacrylate (TMPTMA) a crosslinking agent, were obtained from Aldrich-Chemie (Germany). All monomers were vacuum distilled at $67^{\circ} \mathrm{C}$, under a pressure of $5 \mathrm{~mm} \mathrm{Hg}$ and stored in a refrigerator. The buffers used were, an acetate buffer with acetic acid as buffering agent having $\mathrm{pKa}=4.76$ at $25^{\circ} \mathrm{C}$ and $\mathrm{pH}=4$, the citric acid with $\mathrm{pH}=3$, phosphate and glycin buffers having respectively $\mathrm{pH}=7.4$ and $\mathrm{pH}=10$.

\subsection{Synthesis of hydrogels}

The mixture compositions, as shown in Table 1, were carried out in glass tubes (9 mm I.D., $7 \mathrm{~cm}$ length) and flushed with nitrogen gas for $30 \mathrm{~s}$ to avoid free oxygen. The glass tube was tightly closed and cooled at low temperature 
Table 1. Copolymer gels formulations (volumes are in $\mathrm{ml}$ ). Water content is $1 \mathrm{ml}$ for all gels.

\begin{tabular}{|l|c|c|c|c|}
\hline Gel & HEMA & HEA & DMAEMA & TMPTMA \\
\hline D & 0.90 & & 0.10 & \\
\hline E & & 0.90 & 0.10 & \\
\hline F & 0.85 & & 0.10 & 0.05 \\
\hline G & & 0.85 & 0.10 & 0.05 \\
\hline H & 0.80 & & 0.20 & \\
\hline I & 0.85 & & 0.15 & \\
\hline J & 0.95 & & 0.05 & \\
\hline K & & 0.80 & 0.20 & \\
\hline
\end{tabular}

$-78^{\circ} \mathrm{C}$ with dry ice and acetone, then irradiated by ${ }^{60} \mathrm{Co}$ gamma rays at the dose rate of $22 \mathrm{~Gy} / \mathrm{min}$ to a total dose of $2.5 \mathrm{kGy}$. This irradiation dose enabled almost a total polymerization conversion to be achieved. After irradiation, the cylindrical polymer matrices were cut to thin discs (2-3 mm) and immersed for $24 \mathrm{~h}$ at $4^{\circ} \mathrm{C}$ in phosphate buffer in order to remove the non reacted monomers.

\subsection{Swelling measurements}

The water content, in distilled water at $25^{\circ} \mathrm{C}$, of the hydrogel samples cut into discs of $1.2 \mathrm{~cm}^{2}$ was determined gravimetrically. Samples were weighed after drying the surface, at timed intervals of hydration at room temperature $\left(25^{\circ} \mathrm{C}\right)$. Measurements were taken until equilibrium was reached, when three identical consecutive values were obtained. The degree of hydration $\% \mathrm{H}$ is defined by the following equation

$$
\% \mathrm{H}=\frac{m_{\mathrm{s}}-m_{\mathrm{d}}}{m_{\mathrm{s}}} \times 100
$$

where $m_{s}$ and $m_{d}$ are the weights of the swollen gel at equilibrium and the dry gel, respectively. All swelling studies were carried out in duplicate, and the volume of the buffered solutions was $10 \mathrm{ml}$ in all cases.

\subsection{Scanning Electron Microscopy (SEM)}

Scanning electron microscopy (SEM) analysis was performed on lyophilized samples after swelling. CryoSEM is a typical method to examine the morphology of dried samples of porous hydrogels. It shows that the hydrogels studied have open capillary channels interconnected with each other even when hydrated. They exhibited different pore dimensions as illustrated in Figure 1. In this figure are shown scanning electron micrographs of the surfaces of matrices E, H, I, and J. The polymer wall appeared appeared to be composed of porous network with a spongiform structure. Different morphologies show typical structures due to drying processes such as freezing-drying. Upon placement in fluid, the polymer network hydrates.

\section{RESULTS AND DISCUSSION}

The prepared samples contained a base hydrophilic polymer (HEMA) or (HEA), an amine component

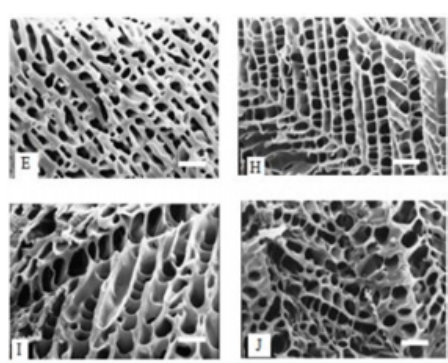

Figure 1. Scanning electron micrographs of the swollen matrices $\mathrm{E}, \mathrm{H}, \mathrm{I}$, and $\mathrm{J}$ immersed in buffer solution at least 1 day and examined using cryoSEM. The scale bars represent $20 \mu \mathrm{m}$.

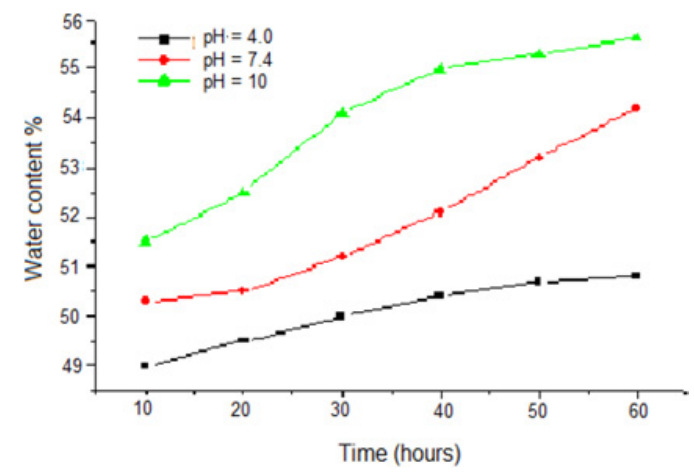

Figure 2. Swelling kinetics of matrix D at $\mathrm{pH} 4,7.4$, and 10 as indicated on the figure.

(DMAEMA), and a cross-linker (TMPTMA) in equal proportion 1:1 (copolymers: distilled water). The total volume was $2 \mathrm{ml}$.

\subsection{Kinetics of swelling}

Swelling kinetics measurements were first performed on disk-shaped (12 mm diameter, $2 \mathrm{~mm}$ thickness) of matrix D which contain essentially HEMA. Results are reported in Figure 2. When a dry matrix is immersed into the solution, it starts to absorb solvent. An increase of water content of hydrogel was observed for low pHs. The equilibrium swelling was reached after 60 hours.

\subsection{Equilibrium degree of swelling}

The swelling of hydrogels as a function of solution $\mathrm{pH}$, chemical nature, percent crosslinking and percent DMAEMA was studied after 60 hours swellingtime. Figure 3 shows the changes in equilibrium water content of different samples. These transition changes according to the external conditions may be defined as stimulisensitivity. The water content depends on the nature of hydrogel andlies between 45 and $74 \%$. Matrices $\mathrm{H}$ and $\mathrm{K}$ containing the same quantity of HEMA, HEA and DMAEMA, present the highest equilibrium water content $72 \%$ and $74 \%$. These high values are due to the presence of $10 \%$ DMAEMA. Matrices $\mathrm{H}$ and I contain $10 \%$ and $7.5 \%$ DMAEMA respectively and show that swelling is more significant for matrix $H$. This can be explained 


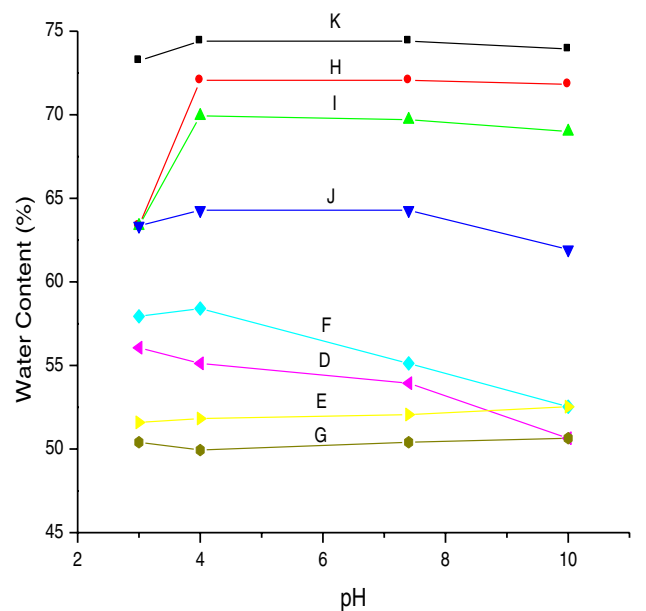

Figure 3. $\mathrm{pH}$ effect on the swelling behaviour of matrices with different compositions.

by the presence of amine groups that become positively charged after protonation in acid medium. The electrostatic repulsion gives more volume to the hydrogel allowing diffusion of higher water content. Similar results were obtained by Kost et al. [20].

\subsection{Effect of the crosslinking agent}

Matrices $F$ and $G$ were prepared by keeping the same concentration of monomers as in matrices $\mathrm{D}$ and $\mathrm{E}$, but by adding a crosslinking agent TMPTMA at $2.5 \%$. The water content of hydrogels based on HEA decreases in acid medium in the presence of TMPTMA. This phenomenon is opposite to the hydrogel based on HEMA. Jim H. Kou et al. [21] reported that the equilibrium state of different polymers is independent of the crosslinker concentration at a low pH. According to Ratner and Miller [22], the swelling of PHEMA is independent of the degree of crosslinking. This is explained by the hydrophobic interaction between the $\alpha$-methyl groups localized on the polymer network. For PHEA, the swelling depends on the degree of crosslinking, because there are no groups $\alpha$ methyl taking part in the hydrophobic interaction [23].

\section{CONCLUSION}

The swelling behavior of hydrogels has been studied extensively in the literature. Hydrogels based on HEMA and HEA have the capacity to absorb a considerable amount of water at different $\mathrm{pH}$ values. It was shown that the water uptake of the hydrogels at the equilibrium state was dependent on the chemical composition of the copolymer and can be influenced by several factors such as the concentration of the charged amine groups and the degree of crosslinking. These results may be of interest in the use of such materials in biomedical applications in the presence of plugs of the physiological fluids. It would be also interesting to study their biocompatibility behavior.

\section{References}

[1] Peppas N.A., Zach Hilt J., Khademhosseini A., Langer R., Adv. Mater, 18, 1345-1360, 2006.

[2] Khorram M., Vasheghani-Farahani E., GolshanEbrahimi N., Iran Polym. J., 12, 315-322, 2003.

[3] Ganji F, Vasheghani-Farahani E., Iran Polym. J.,18, 63-88, 2009.

[4] Hoare T.R., Kohane DS, Polymer, 49, 1993-2007, 2008.

[5] Hosseinkhani H., Hosseinkhani M., Khademhosseini A., Biomaterials, 27, 5836-5844, 2006.

[6] Hosseinkhani H., Hosseinkhani M., Curr. Drug. Saf., 4, 79-83, 2009.

[7] Gao D., Xu H., Philbert M.A., Kopelman R., Angew. Chem. Int. Ed., 46, 2224-2227, 2007.

[8] Aalaie J., Rahmatpour A., Vasheghani-Farahani E., Polym. Adv. Technol., 20, 1102-1106, 2009.

[9] Singh M. et al., Biomed. Eng., 6 (1971) p. 157.

[10] Norihico M., Biocompatiblepolymers/soft tissue compatible polymers, CRC Press Boca Rat (1993), 135.

[11] Tsuruta T., Adv. Polym. Sci. 126:1, 1996.

[12] Hoffman A.S., Adv. Drug. Deliv. Rev. 43:3, 2002.

[13] Kazanskii K.S., Dubrovskii S.A., Adv. Polym. Sci. 104:97, 1992.

[14] Veronese F.M., et al. Controlled Release, 16, 291298, 1991.

[15] Okano T. et al., J. Biomed. Mater.Res. 15, 393, 1981.

[16] Caliceti P., et al., Il Farmaco, 47 (3) 275-286, 1992.

[17] Hoffman A.S., et al., J. Controlled Release, 4, 213-222, 1986.

[18] Yoshida R., et al., Advanced Drug Delivery Reviews, 11, 85-108, 1993.

[19] Vazquez B. et al., Biomaterials, 18, 521-526, 1997.

[20] Kost J. et al., J. Biomed. Mater. Res., 19, 1117-1133, 1985.

[21] Kou J.H. et al., Cosmetic and pharmaceutical applications of polymers, Plenium Press, New York (1991).

[22] Ratner D.B. and Miller I.F., J. Polym. Sci.: Part A-1, 10, 2425, 1972.

[23] Refojo M. et al., J. Polym. Sci., 66, 227, 1979. 